\title{
Medical education in Europe
}

This joint issue of the Netherlands Journal of Medical Education (Tijdschrift voor Medisch Onderwijs) and the German Journal of Medical Education (Zeitschrift für Medizinische Ausbildung) is unique. Both journals are directed at readers who are interested in developments in the area of the education of doctors, dentists, veterinarians and other health care professionals. Both journals primarily present papers and other information dealing with medical education and research that take place in the Dutch-speaking country the Netherlands and in Flanders, the Dutch-speaking region of Belgium or in the German speaking countries Germany, Switzerland and Austria. But nowadays medical education is not confined to national borders. Medical education has become a European concern, or even a broader issue with international impact. Consequently, one might consider the question whether the language of the Tijdschrift voor Medisch Onderwijs and the Zeitschrift für Medizinische Ausbildung should not be changed to English. Clearly, as both journals have different distinguishing features and the countries in which they are published also have their own characteristics, the answers to this question may differ. But, the wish of both Editorial Boards to look beyond national borders was the basis for an experiment: a joint issue of the Tijdschrift voor Medisch Onderwijs and the Zeitschrift für Medizinische Ausbildung in English.

The most relevant example of the European influence on medical education is the Bologna agreement. ${ }^{1}$ In this docu- ment, signed in 1999, European ministers of education agreed that higher education programmes should become organized according to the bachelor-master structure and that the content of programmes should be based on clearly described competencies. The main goal of the Bologna agreement is to stimulate international mobility among students. Naturally, the implementation of the Bologna agreement does not happen identically in all European countries. ${ }^{2}$ In the countries of the Tijdschrift voor Medisch Onderwijs and the Zeitschrift für Medizinische Ausbildung the bachelor-master structure has been implemented in the Netherlands, Flanders and Switzerland, while this change of curriculum design has not yet been adopted in Germany and Austria. Clearly, national or even local traditions and concepts in relation to medical education determine the process of change and explain the great differences in this process. They represent different characteristics of nations, national cultures and professional identities. And, they also may reflect the delicate balance between steps towards an integrated Europe and the conservation of national identity.

When considering publication of a joint issue, the Editorial Boards felt that we should learn from each other. Those who embraced Bologna may realize that it may be attractive to include aspects of the 'old' programmes in the 'new' programmes. And those who keep away from Bologna may realize that this concept also offers great advantages and challenges. In order to compose an attractive 
content of the joint issue, the Boards identified subjects of interest for both groups of readers. Next we invited authors to write a contribution to this special issue. All manuscripts were read by a Dutch and a German speaking reviewer. Their comments and recommendations were incorporated by the authors in the final versions of their manuscripts.

We hope that the content of this joint issue of the Tijdschrift voor Medisch Onderwijs and the Zeitschrift für Medizinische Ausbildung will advance the exchange of experiences between educators, policy makers and other professionals in the field of education for health care professionals. Furthermore, we hope that this issue will help to find an answer to the question about the language of the two journals. But first and foremost, this first issue of the 2010 volume should be considered as a reflection of the great challenges we face in medical education in the Dutch and German speaking countries as well as in Europe.

\section{References}

1. http://www.minocw.nl/bachelor/bolognaverklaring.doc

2. Patricio M, den Engelsen C, Tseng D, ten Cate O. Implementation of the Bologna two-cycle system in medical education. Where do we stand? Med Teach 2008;30:597-605.

Jan Borleffs, Editor in Chief of the Netherlands Journal of Medical Education (Tijdschrift voor Medisch Onderwijs)

Eckhart G. Hahn, Editor in Chief of the German Journal of Medical Education (Zeitschrift für Medizinische Ausbildung) 